\title{
A THEOREM ON POLYNOMIAL IDENTITIES
}

\section{J. LEVITZKI}

1. Introduction. In a recent paper Kaplansky [4 ${ }^{1}$ has emphasized the importance of rings which satisfy a polynomial identity (in short: PI-rings) and proved a number of interesting theorems concerning such rings. In the present note some further properties of PI-rings are derived. In the following, a PI-ring is said to be of degree $d$ if $d$ is the degree of a "minimal equation" (that is, a polynomial identity of minimal degree). In ascribing the term radial to the sum of all nilpotent ideals (notation: $N(S)$ = radical of $S$ ) we may express our main result as follows: The nilpotent elements of a PI-ring ${ }^{2}$ are of bounded index modulo the radical. More precisely: The nilpotent elements of the quotient-ring $S / N(S)$ satisfy the identity $x^{[d / 2]}=0$. This is proved in $\$ 3$ (Theorem 1 ), where also some immediate consequences of this theorem are listed. Extensive use is made of Kaplansky's Lemma 2 [4] which is restated in $\$ 2$ of the present paper (Lemma 3 ) in a slightly generalized form.

In $\$ 4$ we discuss in some detail an application to PI-nil-rings (in short: NPI-rings). Our result in this section is connected with the following construction due to Baer [1]: By means of the transfinite induction he defines the $r$ th radical $N_{r}(S)$ as follows: (1) $N_{1}(S)=N(S)$. (2) If $r=t+1$, then $N_{r}(S)$ is uniquely determined by the relations $N_{r}(S) \supseteq N_{t}(S), N_{r}(S) / N_{t}(S)=N\left[S / N_{t}(S)\right]$. (3) If $r$ is a limit-ordinal, then $N_{r}(S)=U_{t<r} N_{t}(S)$. There exists a smallest ordinal $\lambda$ such that $N_{\lambda}(S)=N_{\lambda+1}(S)$. The ideal $N_{\lambda}(S)$ will be called the ultimate radical ${ }^{3}$ of $S$ and denoted by $U(S)$. For this ideal $N[S / U(S)]$ $=0$, and if for an ideal $A$ the relation $N(S / A)=0$ holds, then $A \supseteq U(S)$. Since also the ideal $N^{*}(S)$, defined as the sum of all seminilpotent ideals, has the property $N\left[S / N^{*}(S)\right]=0$ (see [5]), it follows that $N^{*}(S) \supseteq U(S)$, that is, the ultimate radical is semi-nilpotent.

A ring $T$ which coincides with its ultimate radical will be called an $L$-ring. The smallest ordinal $\lambda$ for which $N_{\lambda}(T)=N_{\lambda+1}(T)$ will be called the length of $T$ (notation: $\lambda=\lambda(T)=$ length of $T$ ). It is an immediate consequence of the definition that each $L$-ring is seminilpotent, and hence a nil-ring.

Presented to the Society, February 26, 1949; received by the editors January 4, 1949.

1 Numbers in brackets refer to the bibliography at the end of the paper.

2 As to the domain of coefficients of the polynomial identities, compare \$2.

3 This ideal was termed by Baer the lower radical. For the purpose of the present note the term ultimate radical seems the more appropriate. 
It has been proved by Kaplansky [4] that an NPI-ring is seminilpotent. In section 4 it is shown that an NPI-ring is an $L$-ring. It should be pointed out in this connection that whereas it is unknown whether or not nil-rings exist which are not semi-nilpotent, it can be deduced from an example due to Baer (see $[1, \S 2]$ ) that a seminilpotent ring need not be an $L$-ring. ${ }^{4}$ We further prove that the length $\lambda(S)$ of an NPI-ring $S$ is finite, and that the degree $d(S)$ is an upper bound for $\lambda(S)$. More precisely: If $S \neq 0$, then $\lambda(S)$ $\leqq \log d(S) / \log 2$. (If $S=0$ then $\lambda=d=1$.) An immediate consequence of Theorem 1 is: If $S$ is an NPI-ring, then the quotient-ring $S / N(S)$ is of bounded index. Moreover, the identity $x^{[d / 2]}=0$ holds in $S / N(S)$.

2. Preliminary remarks. In the case of algebras the coefficients of the polynomial identities are chosen from the underlying field. In order to include this case in our deliberations, we assume that the coefficients $\alpha, \beta, \gamma, \cdots$ of our polynomials belong to a domain $D$ of operators having the following properties:

I. The domain $D$ is a subset of the ring $E$ of endomorphisms of the additive group defined by the given ring $S$.

II. For $\alpha \in D ; s_{1}, s_{2} \in S$, we have $\alpha\left(s_{1} s_{2}\right)=\left(\alpha s_{1}\right) s_{2}=s_{1}\left(\alpha s_{2}\right)$.

III. If $\alpha \in D$, then either $\alpha S=0$ (that is, $\alpha$ is the null-endomorphism) or $\alpha$ is an automorphism. In the latter case we assume that also $\alpha^{-1}$ belongs to $D$.

IV. The domain contains the endomorphisms $0, \pm 1$, and if $\alpha \in D$, then also $-\alpha \in D$.

Each ring possesses an operator domain with properties I-IV, for example, the set of the 3 numbers $0, \pm 1$.

A sub-ring $A$ of $S$ is called $D$-admissible, if for each $\alpha$ we have $\alpha S \subseteq S$.

Lemma 1. If $a \in S$, then $S a, a S$, and $S a S$ are D-admissible.

Proof. For $\alpha(S a)=(\alpha S) a \subseteq S a ; \alpha(a S)=a(\alpha S) \subseteq a S ; \alpha(S a S)=(\alpha S) a S$ $\subseteq S a S$.

Lemma 2. If $A$ is a $D$-admissible sub-ring of $S$ and $0 \neq \alpha \in D$, then $\alpha A=A$.

Proof. For we have $\alpha A \subseteq A=\alpha\left(\alpha^{-1} A\right) \subseteq \alpha A$.

The polynomials which are considered in this paper are elements of the free algebra $E\left(x_{1}, x_{2}, \cdots, x_{n}\right)$ generated (compare [4]) by the

4 This shows incidentally that the ring constructed by Baer furnishes an example of a nil-ring for which no polynomial identity can be found. 
indeterminates $x_{1}, \cdots, x_{n}$ over the ring of endomorphisms $E$; but we restrict our attention only to such polynomials whose coefficients belong to $D$ and whose constant coefficient is 0 . If the polynomial identity

$$
f\left(x_{1}, x_{2}, \cdots, x_{n}\right)=0
$$

is not linear in one of the indeterminates, say $x_{1}$, one performs (see $\left[4\right.$, Lemma 2]) the transformation $g\left(u, t, x_{2}, \cdots, x_{n}\right)$ $=f\left(u+t, x_{2}, \cdots, x_{n}\right)-f\left(u, x_{2}, \cdots, x_{n}\right)-f\left(t, x_{2}, \cdots, x_{n}\right)$. One obtains the identity $g\left(u, t, x_{2}, \cdots, x_{n}\right)=0$ which has a lower degree in $u$ as well as in $t$ than the degree of (1) in $x_{1}$. The general degree of $g$ is not higher than that of $f$, and if the coefficients of $f$ belong to $D$, then this holds also for the coefficients of $g$. If, finally, an indeterminate, say $x_{1}$, does not appear in at least one monomial with a nonzero coefficient, and if $h\left(x_{2}, \cdots, x_{n}\right)$ is the sum of all such monomials, then evidently $h\left(x_{2}, \cdots, x_{n}\right)=0$ holds in $S$. As a consequence of these remarks we have the following lemma.

LEMma 3. If $S$ is a PI-ring of degree d, then $S$ satisfies a polynomial identity of the form

$$
f\left(x_{1}, x_{2}, \cdots, x_{d}\right)=\sum_{(i) \in \Pi} \alpha_{(i)} x_{i 1} \cdots x_{i d}=0
$$

where $\Pi$ is a set of permutations $(i)$ of the $d$ numbers $1,2, \cdots, d$ and $\alpha_{(i)} \in D$.

3. The main theorem. We assume in this section that the ring $S$ has an operator domain $D$ satisfying conditions I-IV of $\$ 2$ and that the coefficients of all polynomial identities belong to this domain.

Theorem 1. If $S$ is a $P I$-ring of degree $d$ and $N(S)$ the radical of $S$, then for each nilpotent element a of $S$ we have

$$
a^{[d / 2]} \in N(S) \text {. }
$$

Proof. This is true for $a \in N(S)$. Now suppose that $a \notin N(S)$, and denote by $n$ the index of $a$ modulo $N(S)$, that is,

$$
a^{n} \notin N(S), \quad a^{n+1} \in N(S) .
$$

The theorem will be proved if we show that $n<[d / 2]$. To this end consider the following $2 n+1$ sub-rings of $S$

$$
\begin{aligned}
A_{2 j-1} & =a^{n-j+1} S a^{j-1}, & j=1,2, \cdots, n+1, \\
A_{2 j} & =a^{n-j+1} \mathrm{Sa}^{j}, & j=1,2, \cdots, n,
\end{aligned}
$$


and form the products

$$
B_{i}=A_{1} \cdot A_{2} \cdots A_{i}, \quad i=1,2, \cdots, 2 n+1 .
$$

It follows that

$$
\begin{aligned}
B_{2 j-1} & =\left(a^{n} S\right)^{2 j-1} a^{j-1}, & j=1,2, \cdots, n+1, \\
B_{2 j} & =\left(a^{n} S\right)^{2 j} a^{j}, & j=1,2, \cdots, n .
\end{aligned}
$$

From (5) we deduce that $A_{s} A_{t} \subseteq S a^{n+1} S$ for $s>t$. Hence if for any integer $r$ such that $r \leqq 2 n+1$ one denotes by $\left(i_{1}, \cdots, i_{r}\right)$ a permutation of the integers $1,2, \cdots, r$, we have

$$
A_{i_{1}} A_{i_{2}} \cdots A_{i_{r}} \subseteq S a^{n+1} S \quad \text { if }\left(i_{1} \cdots i_{r}\right) \neq(1, \cdots, r)
$$

or in other words

$$
\begin{gathered}
a_{i_{1}} a_{i_{2}} \cdots a_{i_{r}} \in S a^{n+1} S \\
\text { for } a_{k} \in A A_{k}, k=1, \cdots, r \text { and }\left(i_{1}, \cdots, i_{r}\right) \neq(1, \cdots, r) .
\end{gathered}
$$

We turn now to the given polynomial identity which according to Lemma 3 we may write in the form

$$
\beta x_{1} x_{2} \cdots x_{d}=\sum_{(i) \in \mathbb{I},} \beta_{(i)} x_{i_{1}} \cdots x_{i d}, \quad \beta \neq 0,
$$

where the permutations $(i)$ of the set $\Pi^{\prime}$ are all different from the identical permutation. Suppose now that $n \geqq[d / 2]$; then we have $n \geqq(d-1 / 2)$ or $d \leqq 2 n+1$. Hence we may substitute in (9) the integer $d$ for $r$, thus obtaining

$$
a_{i_{1}} a_{i_{2}} \cdots a_{i_{d}} \in S a^{n+1} S \quad \text { for }\left(i_{1} \cdots i_{d}\right) \neq(1, \cdots, d) .
$$

Since $\beta_{(i)} S a^{n+1} S \subseteq S a^{n+1} S$ (Lemma 1) we obtain in view of ${ }^{6}(10)$

$$
\beta a_{1} a_{2} \cdots a_{d} \in S a^{n+1} S
$$

or $\beta A_{1} \cdots A_{d}=\beta B \subseteq S a^{n+1} S$. By formulas (7) we now have

$$
\begin{array}{lrl}
\beta B_{d}=\beta\left(a^{n} S\right)^{2 q-1} a^{q-1} \subseteq S a^{n+1} S, & \text { if } d=2 q-1, \\
\beta B_{d}=\beta\left(a^{n} S\right)^{2 q} a^{q} \subseteq S a^{n+1} S & \text { if } d=2 q .
\end{array}
$$

Right multiplication of the first relation in (13) by $a^{n-q+1} S a^{n} S$, and

- A slight simplification of the argument is possible by operating in the quotientring $S / S a^{n+1} S$. We have then zero on the right side of formulas (8), (9), and (11)-(14). Passage to this quotient-ring is permissible since the ideal $S a^{n+1} S$ is admissible. This ensures the survival of the polynomial identity and, in view of postulate III, also of its degree; each nonzero endomorphism of $S$ becomes a nonzero endomorphism of $S / S a^{n+1} S$. 
of the second by $a^{n-q} S$, yields in both cases in view of $\beta\left(a^{n} S\right)=a^{n} S$ (Lemmas 1 and 2)

$$
\left(a^{n} S\right)^{2 q+1} \subseteq S a^{n+1} S .
$$

Now we assumed in (4) that $a^{n+1} \in N(S)$, which implies that the ideal $S a^{n+1} S$ is nilpotent. For some integer $t$ we have therefore $\left(a^{n} S\right)^{t(2 q+1)}$ $=0$, which implies that $a^{n} \in N(S)$, contradicting (4). We were led to this contradiction by assuming that $n \geqq[d / 2]$. Hence $n<[d / 2]$, q.e.d.

Consequences. (1) Let $S$ denote a PI-ring of degree $d$, and $T$ a $D$ homomorphic image of $S$. If $N(T)=0$, then the nilpotent elements of $T$ are of bounded index. Moreover, these elements satisfy the identity $x^{[d / 2]}=0$. Indeed, the polynomial identity satisfied by $S$ as well as its degree are inherited by $T$, and our assertion is therefore a direct consequence of Theorem 1 .

(2) If $S$ is the ring of $n$ by $n$ matrices over a commutative or a noncommutative field $F$, and if $d$ is the degree of a polynomial identity satisfied by $S$, then $n \leqq[d / 2]$. Indeed, this is a consequence of $N(S)=0$ and of the fact that $S$ contains nilpotent elements of index $n-1$. This implies Kaplansky's Lemma 5 [4] which he applies in the proof of his Theorem 1 which states that each PI-primitive algebra in the sense of Jacobson [3] is finite-dimensional over its center. As to the problem raised by Kaplansky concerning an explicit upper bound for the order of the algebra over its center, our exponent $[d / 2]$ yields a rather sharp estimate. By using Kaplansky's Lemma $3[4]$ we have the following consequence.

(3) If $k^{2}$ is the order of a simple algebra over its center, and $d$ is the degree of a polynomial identity, then we have

$$
k^{2} \leqq[d / 2]^{2} \text {. }
$$

(4) In case $d=1$ the situation is trivial $(S=0)$. Also the case $d=2$, which is a slight generalization of the commutative case, yields nothing new. We have here $[d / 2]=1$, that is, all nilpotent elements belong to $N(S)$, and if $S$ is an algebra of finite order, then $S / N(S)$ is in view of (3) a direct sum of commutative fields.

(5) In case $d=3$ we have $[d / 2]=1$, that is, again all nilpotent elements of $S$ belong to $N(S)$. It is of interest to note that just as in the commutative case we have also here the coincidence of all radicallike ideals which have been hitherto defined by using various types of nillity. If $S$ is an algebra of finite order it follows in view of (3) that $S / N(S)$ is the direct sum of commutative fields, a fact which was also mentioned by Kaplansky. 
(6) In case $d=4$ or $d=5$ we have $[d / 2]=2$. If $S$ is a division algebra which satisfies a polynomial identity of degree 4 or 5 , it follows in view of (3) that $S$ is either a commutative field or a generalized quaternion algebra. This was previously proved by Hall [2, Theorem 6.2] for the case of a specific polynomial identity of degree 5. Compare also [4].

(7) A direct simple proof of statement (2) may be deduced as follows: Consider the $n^{2}$ matrices $c_{i j}, i, j=1, \cdots, n$, satisfying the relations

$$
c_{i j} c_{j k}=\left\{\begin{array}{lll}
0 & \text { if } & j \neq j^{\prime} \\
c_{i k} & \text { if } & j=j^{\prime}
\end{array}\right.
$$

Put $a_{2 i-1}=c_{i i}, a_{2 i}=c_{i i+1}$ and form the product $a_{1} a_{2} \cdots a_{r}$ for each positive integer $r<2 n$. Suppose that $d(S)<2 n$ and write the polynomial identity in the form (10). By substituting $a_{i}$ for $x_{i}$, $i=1, \cdots, d$, one obtains zero on the right side of (10) and something not equal to 0 on the left. This contradiction is a consequence of $d<2 n$. Hence $d \geqq 2 n$, q.e.d.

4. Polynomial identities and $L$-rings. Also in this section we assume that all polynomial identities considered have their coefficients in a domain $D$ as defined in $\$ 2$.

THEOREM 2. If $S$ is an NPI-ring of degree d, and $N_{r}(S)$ is the rth radical of $S$, then the ring $S / N_{r}(S)$ satisfies the identity

$$
x^{[d / 2]}=0 .
$$

Proof. For $r=1$ this is an immediate consequence of Theorem 1. For $r>1$ this follows from $N_{r}(S) \supseteq N_{1}(S)$.

Theorem 3. If $S$ is an NPI-ring and $S \supset 0$, then also $N(S) \supset 0 .^{6}$

Proof. If $d$ is the degree of $S$, then for each $x \in S$ we have by Theorem 1 the relation $x^{[d / 2]} \in N(S)$. From $S \neq 0$ it follows that $d \geqq 2$. In case $2 \leqq d \leqq 3$, we have $[d / 2]=1$, that is, $x \in N(S)$ or $S=N(S)$, and the theorem is proved. We consider now the case $d \geqq 4$ and suppose that $N(S)=0$. It follows then that the identity $x^{[d / 2]}=0$ whose degree is smaller than $d$ holds in $S$, which is a contradiction. Hence $N(S) \neq 0$, q.e.d.

Theorem 4. Each NPI-ring is an L-ring.

Proof. By Theorem 2 the quotient-ring $S / N(S)$ satisfies an iden-

- This theorem is in some respect a generalization of the fact that for each commutative nil-ring we have $N(S)=S$. 
tity of the form $x^{p}=0$. Since $U(S) \supseteq N(S)$, this identity is satisfied also in $S / U(S)$. In view of $U[S / U(S)]=0$ we have also $N[S / U(S)]$ $=0$ and hence it follows by Theorem 3 that $S / U(S)=0$, or $S=U(S)$, q.e.d.

Corollary. Denote by $S$ an arbitrary $P I$-ring. If $U(S)$ is the ultimate radical and $U^{\prime}(S)$ is the upper radical (see Baer [1]), then: (1) $U(S)=U^{\prime}(S)$ and (2) the quotient-ring $S / U(S)$ has no one-sided nil-ideals other than zero.

Indeed, since $U(S)$ is $D$-admissible (see [1]) the polynomial identity, and in view of postulate III in $\$ 2$ also its degree are inherited by $U^{\prime}(S) / U(S)$. Since $N\left[U^{\prime}(S) / U(S)\right]=0$ (compare [1]) it follows by Theorem 3 that $U^{\prime}(S) / U(S)=0$, or $U^{\prime}(S)=U(S)$. To prove the second part of the above statement, suppose that $R$ is a right (left) nil-ideal in $S$. By Theorem 4 it follows that $R$ is an $L$-ring, and hence semi-nilpotent. We have therefore $R \subseteq U^{*}(S)$, where $U^{*}(S)$ is the sum of all semi-nilpotent ideals of $S$. Since $U^{*}(S) \subseteq U^{\prime}(S)$ $=U(S)$, we have $R \subseteq U(S)$, which implies the second part of our statement.

TheOREM 5. Let $S$ be an NPI-ring of degree $d_{0}, N_{r}(S)$ the rth radical of $S$, and $d_{r}$ the degree of the quotient-ring $S_{r}=S / N_{r}(S)$. Then:

(1) If $d_{r}>1$ then $d_{r+1} \leqq d_{r} / 2$.

(2) The ring $S$, which by Theorem 4 is an L-ring, has finite length.

(3) If $\lambda=\lambda(S)$ denotes the length of $S$, then

$$
\lambda \leqq \log d_{0} / \log 2 \text { for } d_{0}>1 \text {. If } d_{0}=1 \text {, then } \lambda=1 \text {. }
$$

Proof. (1) For $r=0$ this is an immediate consequence of Theorem 1. Now assume $r>0$. We know (for example, by Theorem 2) that $S_{r}$ satisfies a polynomial identity. If $d_{r}$ is the degree of $S_{r}$, then by Theorem 2 the ring $S_{r} / N\left(S_{r}\right)$ satisfies the identity

$$
x^{\left[d_{r} / 2\right]}=0 .
$$

By definition $N\left(S_{r}\right)=N_{r+1}(S) / N_{r}(S)$ and therefore $S_{r} / N\left(S_{r}\right)$ $=\left[S / N_{r}(S)\right] /\left[N_{r+1}(S) / N_{r}(S)\right] \cong S / N_{r+1}(S)=S_{r+1}$. This isomorphism implies that identity (18) is satisfied by $S_{r+1}$. If now $d_{r+1}$ is the degree of $S_{r+1}$, we have therefore $d_{r+1} \leqq\left[d_{r} / 2\right] \leqq d_{r} / 2$, q.e.d.

(2) Suppose that $d_{r}>1$ for each finite $r$; then by (1) we would obtain an infinite sequence $d_{1}>d_{2}>\ldots$, a contradiction. Hence there exists a finite index $j$ so that $d_{j}=1$. This means that the ring $S / N_{j}(S)$ satisfies an identity of degree 1 , and hence $S / N_{j}(S)=0$, or $S=N_{j}(S)$. By the definition of length we have therefore $\lambda \leqq j$, q.e.d. 
(3) If $d_{0}=1$, then $0=S=N_{1}(S)$, or $\lambda=1$ in which case (3) is proved. If $d_{0}>1$ and $\lambda=1$, then (3) is also true. Now suppose that $\lambda>1$, then evidently $d_{r}>1$ for $r \leqq \lambda-1$ (otherwise we would have $S=N_{r}(S)$ for some $r$ which is smaller than $\lambda$, contradicting the definition of length). This implies in view of (1) that $d_{\lambda} \leqq d_{0} / 2^{\lambda}$ or $2^{\lambda} \leqq d_{0} / d_{\lambda}$, that is, $\lambda \log 2 \leqq \log d_{0}-\log d_{\lambda} \leqq \log d_{0}$, q.e.d.

COROLLARY. The length of an NPI-ring is smaller than the degree of the identity. Eguality holds only in the trivial case $S=0$.

\section{BIBLIOGRAPHY}

1. R. Baer, Radical ideals, Amer. J. Math. vol. 65 (1943) pp. 537-568.

2. M. Hall, Projective planes, Trans. Amer. Math. Soc. vol. 54 (1943) p. 537.

3. N. Jacobson, Structure theory for algebraic algebras of bounded degree, Ann. of Math. (2) vol. 46 (1945) pp. 695-707.

4. I. Kaplansky, Rings with a polynomial identity, Bull. Amer. Math. Soc. vol. 54 (1948) pp. 575-580.

5. J. Levitzki, On the radical of a general ring, Bull. Amer. Math. Soc. vol. 49 (1943) pp. 461-466.

HEBREW UNIVERSITY 